\title{
Signet Ring Cell Carcinoma Mimicking Gastric Gastrointestinal Stromal Tumor: A Case Report
}

\author{
Jin Lee ${ }^{a}$ Sung Jin Oh ${ }^{b}$ \\ aDepartment of Internal Medicine, Inje University Haeundae Paik Hospital, Inje University \\ College of Medicine, Busan, Republic of Korea; bepartment of Surgery, Inje University \\ Haeundae Paik Hospital, Inje University College of Medicine, Busan, Republic of Korea
}

\section{Keywords}

Submucosal tumor · Signet ring cell carcinoma - Gastrointestinal stromal tumors

\begin{abstract}
The presentation of submucosal gastric cancer, especially signet ring cell carcinoma, is rare. The submucosal tumor (SMT) is covered with normal mucosa, and confirmation is difficult through endoscopic biopsy; thus, histologic diagnosis is important to determine the appropriate treatment method. Here, we report a case of gastric signet ring cell carcinoma mimicking gastrointestinal stromal tumor (GIST). A 2-cm-sized SMT suspected of being a GIST on preoperative endoscopic ultrasonography and computed tomography was suspected to be cancerous during surgery. The frozen diagnosis of the resected mass (obtained via wedge resection) showed a carcinoma with signet ring feature, and additional frozen diagnosis of one enlarged lymph node revealed metastatic cancer. Therefore, subtotal gastrectomy with lymph node dissection was performed. This case report suggests that preoperative histologic diagnosis of high-risk SMT might be useful, although the tumor had typical features of GIST on preoperative imaging. Overall, if a tumor is suspected of being a gastric carcinoma during surgery and the differential diagnosis between gastric carcinoma and GIST is uncertain, a careful surgical approach should be considered to account for the possibility of adenocarcinoma.

\section{Introduction}

Gastric cancer with a submucosal tumor (SMT) is rare [1]; as the surface of the tumor is covered with normal mucosa, histologic diagnosis of the lesion is difficult. However, it is important to determine the appropriate treatment method for these patients. Various 

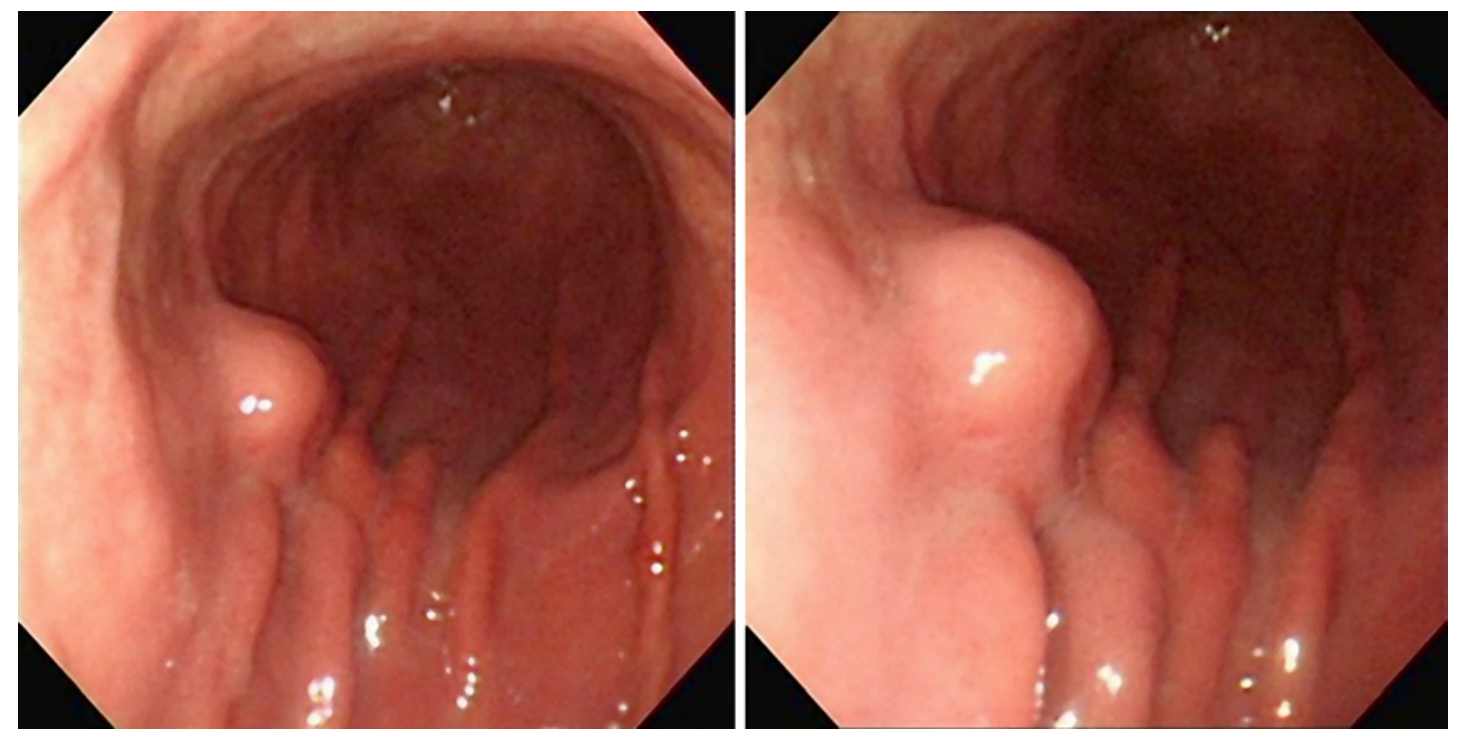

Fig. 1. Endoscopy showing a 2-cm lobulated lesion with normal mucosa at the anterior wall of the mid body.

methods including endoscopic ultrasound-guided fine needle aspiration (EUS-FNA) [2-4], EUS-guided trucut biopsy (EUS-TCB) [5-7], endoscopic submucosal dissection or mucosal resection [8-10], and surgery are widely used for histologic confirmation. In many cases, surgical resection, such as laparoscopic wedge resection, is considered the first method of treatment for SMT because simultaneous diagnosis and treatment are possible. However, in some cases, the tumor may be diagnosed as gastric cancer during or after wedge resection of SMT. Herein, we report a case of signet ring cell type advanced gastric cancer that mimicked gastrointestinal stromal tumor (GIST), diagnosed during laparoscopic wedge resection and treated with subtotal gastrectomy with lymph node dissection.

\section{Case Presentation}

A 64-year-old woman was referred for further evaluation of suspicious SMT. Endoscopy showed an approximately 2-cm-sized lobulated lesion with normal mucosa at the anterior wall of the mid body (Fig. 1). EUS with a linear endoscope (UM-3R miniprobe; Olympus, Tokyo, Japan) was performed to assess the characteristics of the lesion. EUS showed an approximately $19 \times 11 \mathrm{~mm}$ heterogeneous hypoechoic lesion with a well-circumscribed margin that was thought to arise from the fourth layer (muscularis propria) (Fig. 2). However, the mucosal and submucosal layers were intact. Furthermore, EUS with a radial endoscope (UE 260; Olympus, Tokyo, Japan) was subsequently performed and showed no lymph node enlargement. Three-dimensional computed tomography (CT) of the stomach revealed a 2-cm subepithelial lesion with delayed enhancement in the anterior wall of the gastric mid body (Fig. 3). There was no definite evidence of lymph node enlargement or other metastases. GIST was clinically suspected based on the CT and EUS results.

Histologic confirmation or resection was considered because the tumor size was approximately $2 \mathrm{~cm}$. Laparoscopic wedge resection was planned for histologic confirmation and treatment after consultation with the patient. During the surgery in November 2019, a tumor, approximately $3.5 \mathrm{~cm}$ in size, was observed on the anterior wall of the mid body of the stomach, which showed a suspected gastric cancer invading the serosa. After wedge resection, 
Oncology
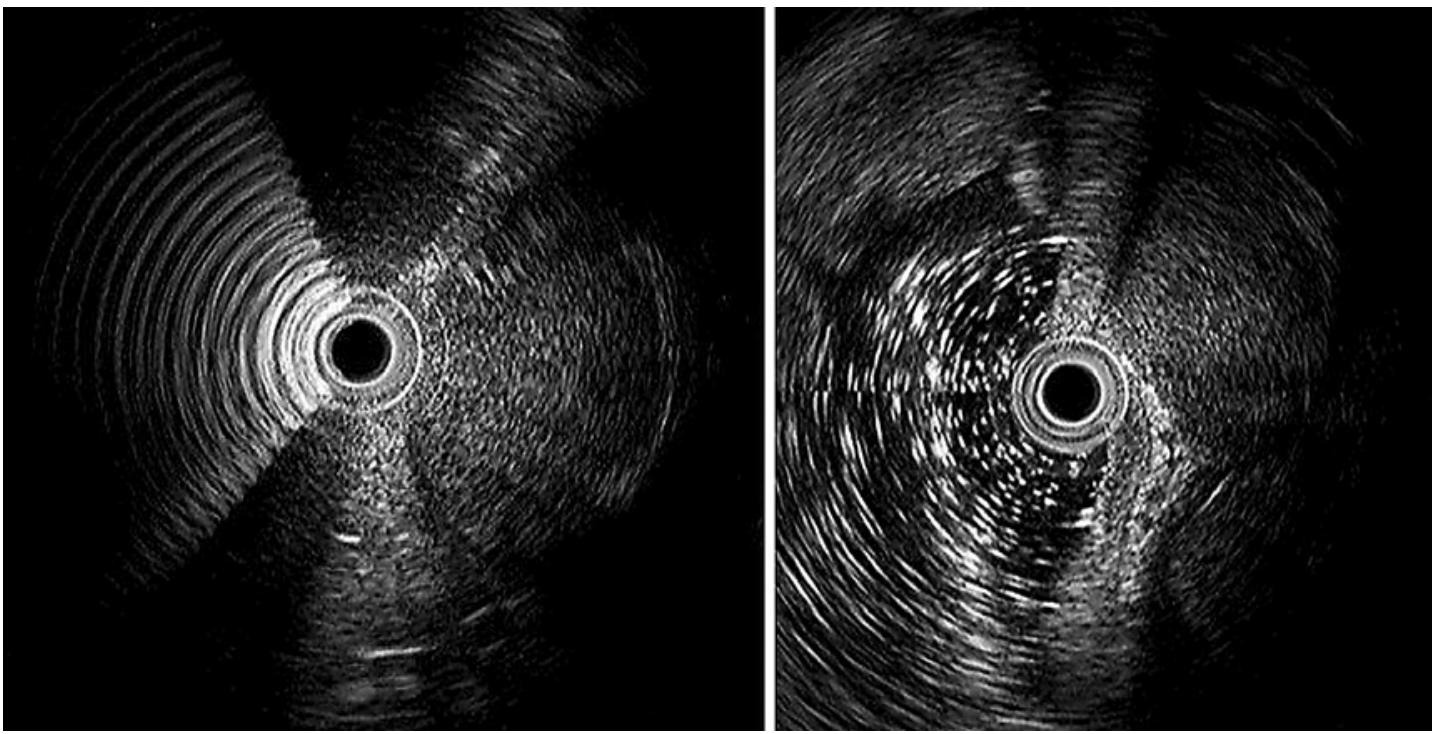

Fig. 2. Endoscopic ultrasound shows an approximately $19 \times 11 \mathrm{~mm}$ heterogeneous hypoechoic lesion with a well-circumscribed margin.
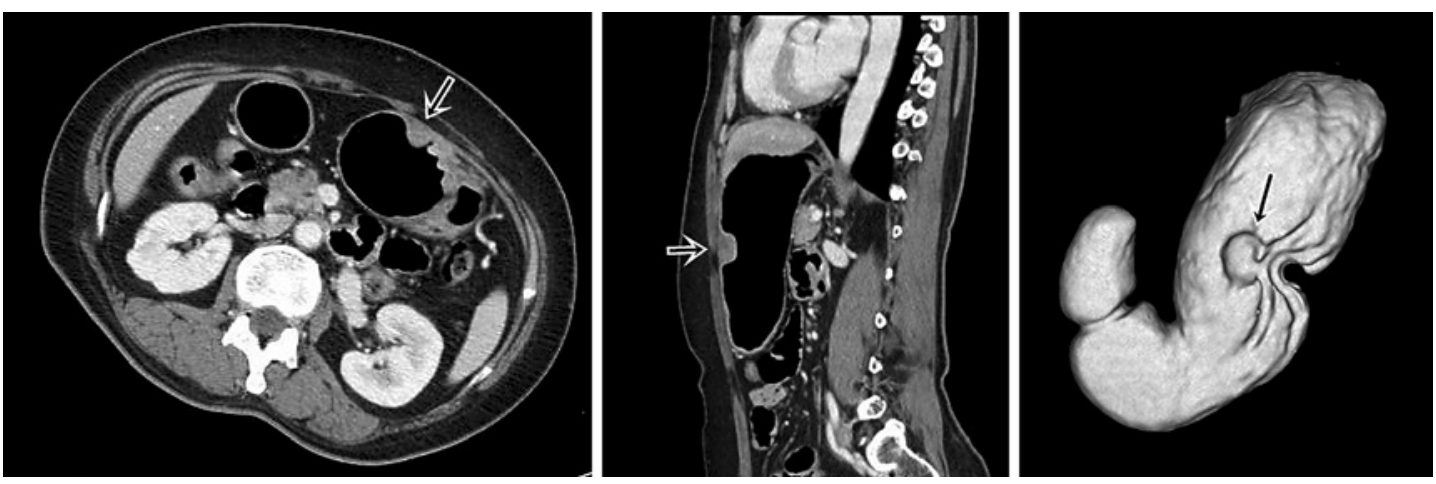

Fig. 3. Stomach 3D computed tomography revealing a 2-cm subepithelial lesion with delayed enhancement (arrow) in the anterior wall of the gastric mid body.

frozen biopsy was performed for the resected mass lesion and demonstrated a carcinoma with signet ring features. An approximately $1.5-\mathrm{cm}$-sized solitary enlarged lymph node was observed below the greater curvature, and the frozen diagnosis revealed metastatic cancer. Radical subtotal gastrectomy with Billroth II was subsequently performed after confirming the frozen diagnosis. Gross examination showed an ulcerative lesion measuring $3.5 \times 3.0 \mathrm{~cm}$, and multiple cut sections revealed a grayish white mass infiltrating the serosal layer. The gross tumor type was defined as Borrmann type 3. Histopathological analysis revealed that the tumor had invaded the serosa (T4a stage) and was a signet ring cell carcinoma with regional metastasis to 7 of 27 lymph nodes (N3 stage) (Fig. 4). The final diagnosis was a signet ring cell type gastric carcinoma with regional lymph node metastasis.

The patient received chemotherapy with capecitabine and intravenous oxaliplatin after recovering from surgery. Subsequent follow-up CT and upper gastrointestinal endoscopy were scheduled 6 months after surgery for the patient. 

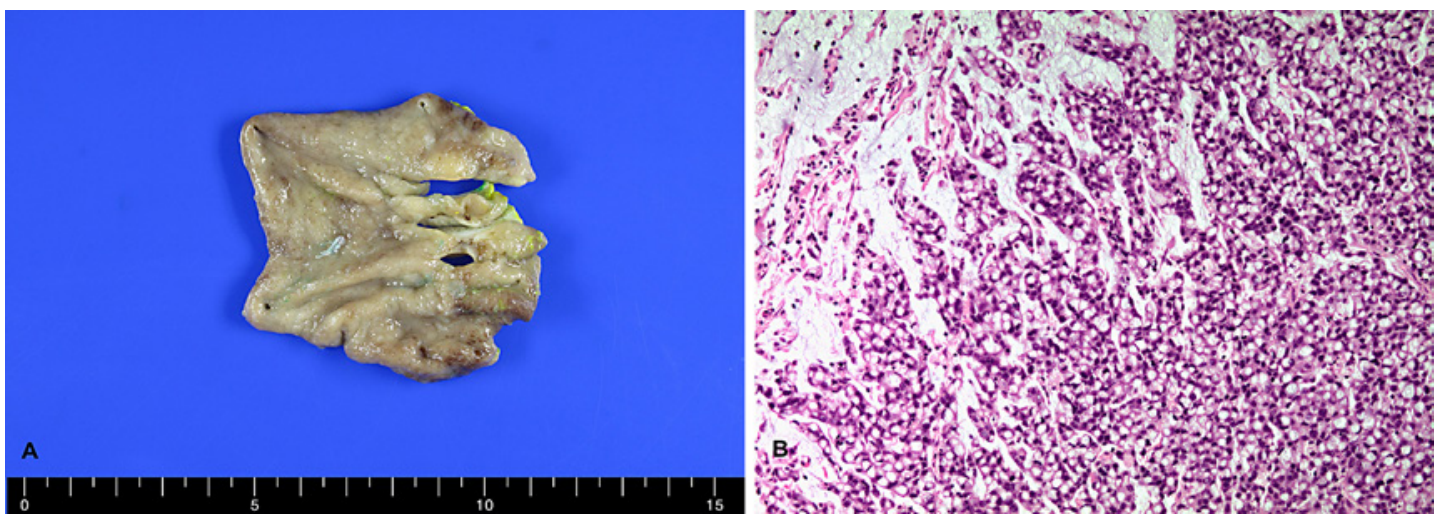

Fig. 4. A Gross examination showing an ulcerative lesion measuring $3.5 \times 3.0 \mathrm{~cm}$, and multiple cut sections revealing a grayish white mass infiltrating the serosal layer. B Numerous infiltrating signet ring cells are noted; H\&E stain, $\times 200$.

\section{Discussion and Conclusion}

Gastric cancer mimicking SMT is very rare; it accounts for $0.1-0.63 \%$ of all resected gastric cancers [1]. Most gastric cancers can be diagnosed through routine endoscopic biopsy. However, a gastric cancer mimicking SMT is difficult to confirm histologically because the tumor surface is covered by normal mucosa and can mimic an SMT, such as GIST or leiomyoma, in terms of endoscopic findings.

Asymptomatic gastric SMTs are frequently found by routine endoscopy, and GISTs account for half of these gastric SMTs [11]. Most GISTs appear as SMT on endoscopy and are often confirmed by surgical resection. If an SMT is less than $2 \mathrm{~cm}$ in size or does not have highrisk features, it is recommended that regular follow-up endoscopy be performed [12-14]. High-risk features include irregular borders, ulcers on the surface, internal heterogeneity or regional lymph node enlargement on EUS, and increasing size during follow-up.

Differential diagnosis between GIST and gastric cancer mimicking SMT is important in clinical practice because of the differences in surgical methods. In the treatment of locally advanced or borderline resectable GIST, wedge resection is performed while regional lymph node dissection is not performed because lymphatic metastasis is rare. Unlike surgery for GISTs, gastric cancer requires a gastrectomy with lymph node dissection.

EUS can provide useful information on SMT, and the need for endoscopic diagnosis or therapeutic approaches can be based on EUS features of the tumors. However, histologic confirmation of lesions is difficult using EUS alone. Various methods, such as EUS-FNA, EUS-TCB, endoscopic submucosal dissection or mucosal resection, laparoscopic excision biopsy, and surgery are used to confirm the histologic diagnosis [2-10].

EUS-FNA or EUS-TCB is a useful method for histologic diagnosis of SMTs before surgery [2-7]. Although EUS-FNA or EUS-TCB is a reliable diagnostic method for SMTs, its sampling adequacy and diagnostic rates have been reported to be $74-83 \%$ and $71-83 \%$, respectively [2-7]. The diagnostic accuracy of EUS-FNA or TCB is influenced by many factors, including features of a mass lesion, size of the needle used, and degree of technical difficulty of the procedure. Considering the various diagnostic yields and high costs of EUS-FNA or EUS-TCB, the best method for histologic confirmation has not yet been established. Economic factors may be important; thus, the cost-benefit ratio should be considered in the selection of treatment methods. In many cases, surgical resection, such as laparoscopic wedge resection, is considered as the first treatment method for SMT because simultaneous diagnosis and 
Lee and Oh: SRC Mimicking Gastric GIST

treatment are possible. However, in some cases, the tumor may be diagnosed as gastric cancer during or after wedge resection of SMT.

Overall, this case suggests that preoperative histologic diagnosis of high-risk SMT might be useful, although the tumor has the typical features of GIST on preoperative imaging. If a histologically undiagnosed SMT is suspected of being a gastric cancer during surgery and the differential diagnosis of gastric cancer and GIST is uncertain, a careful surgical approach is required considering the possibility of adenocarcinoma.

\section{Statement of Ethics}

Written informed consent for the publication of the patient's clinical details and images was obtained from the patient.

\section{Disclosure Statement}

The authors have no conflicts of interest to declare.

\section{Funding Sources}

The authors declared that this study has received no financial support.

\section{Author Contributions}

Jin Lee drafted the manuscript. Sung Jin Oh revised the manuscript. Jin Lee and Sung Jin Oh were involved in the conception and design of the case report.

\section{References}

1 Umehara Y, Kimura T, Okubo T, Sano Y, Nakai K, Oi S, et al. Gastric carcinoma resembling submucosal tumor. Gastric Cancer. 1999;2:191-3.

2 Mekky MA, Yamao K, Sawaki A, Mizuno N, Hara K, Nafeh MA, et al. Diagnostic utility of EUS-guided FNA in patients with gastric submucosal tumors. Gastrointest Endosc. 2010;71:913-9.

3 Sepe PS, Moparty B, Pitman MB, Saltzman JR, Brugge WR. EUS-guided FNA for the diagnosis of GI stromal cell tumors: sensitivity and cytologic yield. Gastrointest Endosc. 2009;70:254-61.

4 Philipper M, Hollerbach S, Gabbert HE, Heikaus S, Böcking A, Pomjanski N, et al. Prospective comparison of endoscopic ultrasound-guided fine-needle aspiration and surgical histology in upper gastrointestinal submucosal tumors. Endoscopy. 2010;42:300-5.

5 Fernandez-Esparrach G, Sendino O, Sole M, Pellisé M, Colomo L, Pardo A, et al. Endoscopic ultrasound-guided fine-needle aspiration and trucut biopsy in the diagnosis of gastric stromal tumors: a randomized crossover study. Endoscopy. 2010;42:292-9.

6 Hoda KM, Rodriguez SA, Faigel DO. EUS-guided sampling of suspected GI stromal tumors. Gastrointest Endosc. 2009;69:1218-23.

7 Polkowski M, Gerke W, Jarosz D, Nasierowska-Guttmejer A, Rutkowski P, Nowecki ZI, et al. Diagnostic yield and safety of endoscopic ultrasound-guided trucut [corrected] biopsy in patients with gastric submucosal tumors: a prospective study. Endoscopy. 2009;41:329-34.

8 Lee I, Lin P, Tung S, Shen C, Wei K, Wu C. Endoscopic submucosal dissection for the treatment of intraluminal gastric subepithelial tumors originating from the muscularis propria layer. Endoscopy. 2006;38:1024-8.

9 Lee CK, Chung I-K, Lee S-H, Lee SH, Lee TH, Park SH, et al. Endoscopic partial resection with the unroofing technique for reliable tissue diagnosis of upper GI subepithelial tumors originating from the muscularis propria on EUS (with video). Gastrointest Endosc. 2010;71:188-94. 
Lee and Oh: SRC Mimicking Gastric GIST

10 Lee HL, Kwon OW, Lee KN, Jun DW, Eun CS, Lee OY, et al. Endoscopic histologic diagnosis of gastric GI submucosal tumors via the endoscopic submucosal dissection technique. Gastrointest Endosc. 2011;74:693-5.

11 Kawanowa K, Sakuma Y, Sakurai S, Hishima T, Iwasaki Y, Saito K, et al. High incidence of microscopic gastrointestinal stromal tumors in the stomach. Hum Pathol. 2006;37:1527-35.

12 Nishida T, Blay JY, Hirota S, Kitagawa Y, Kang YK. The standard diagnosis, treatment, and follow-up of gastrointestinal stromal tumors based on guidelines. Gastric Cancer. 2016;19(1):3-14.

13 Cho JW. Current guidelines in the management of upper gastrointestinal subepithelial tumors. Clin Endosc. 2016;49:235-40.

14 Demetri GD, Benjamin RS, Blanke CD, Blay JY, Casali P, Choi H, et al. NCCN Task Force report: management of patients with gastrointestinal stromal tumor (GIST): update of the NCCN clinical practice guidelines. J Natl Compr Canc Netw. 2007;5(Suppl 2):S1-S29. 17624289. 